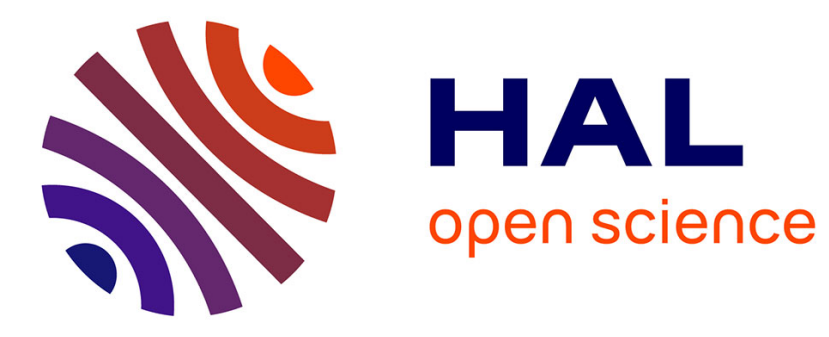

\title{
Experimental and theoretical progress in pipe flow transition
}

\author{
A.P Willis, Jorge Peixinho, R.R R Kerswell, T. Mullin
}

\section{To cite this version:}

A.P Willis, Jorge Peixinho, R.R R Kerswell, T. Mullin. Experimental and theoretical progress in pipe flow transition. Philosophical Transactions of the Royal Society A: Mathematical, Physical and Engineering Sciences, 2008, 366 (1876), pp.2671-2684. 10.1098/rsta.2008.0063 . hal-02143002

\section{HAL Id: hal-02143002 \\ https://hal.science/hal-02143002}

Submitted on 29 May 2019

HAL is a multi-disciplinary open access archive for the deposit and dissemination of scientific research documents, whether they are published or not. The documents may come from teaching and research institutions in France or abroad, or from public or private research centers.
L'archive ouverte pluridisciplinaire HAL, est destinée au dépôt et à la diffusion de documents scientifiques de niveau recherche, publiés ou non, émanant des établissements d'enseignement et de recherche français ou étrangers, des laboratoires publics ou privés. 


\title{
Experimental and theoretical progress in pipe flow transition.
}

\author{
By A.P. Willis*, J. Peixinho ${ }^{\dagger}$, R.R. Kerswell* \& T. Mullin ${ }^{\dagger}$ \\ * School of Mathematics, University of Bristol, UK. \\ $\dagger$ Manchester Centre for Nonlinear Dynamics, University of Manchester, UK.
}

There have been many investigations of the stability of Hagen-Poiseuille flow in the one hundred and twenty five years since Osborne Reynolds' famous experiments on the transition to turbulence in a pipe, and yet the pipe problem remains the focus of attention of much research. Here we discuss recent results from experimental and numerical investigations obtained in this new century. Progress has been made on three fundamental issues: the threshold amplitude of disturbances required to trigger a transition to turbulence from the laminar state, the threshold Reynolds number flow below which a disturbance decays from turbulence to the laminar state, with quantitative agreement between experimental and numerical results, plus understanding the relevance of recently-discovered families of unstable travelling wave solutions to transitional and turbulent pipe flow.

Keywords: pipe, transition, numerical, experimental

\section{Introduction}

The stability of Hagen-Poiseuille flow (Hagen 1839, Poiseuille 1840) in a long circular pipe has intrigued scientists ever since Reynolds' (1883) original experiments. Reynolds showed that the single control parameter for the flow is what is now called the Reynolds number, $R e:=U D / \nu$ where $U$ is the mean velocity, $D$ is the pipe diameter, and $\nu$ the kinematic viscosity of the fluid. His research was mainly focused on transition initiated at the entrance to the pipe, and an important aspect of his work was in showing the importance of controlling entry conditions. The majority of the subsequent experimental investigations of this problem have concentrated on the effects of disturbances created at the inlet, as reviewed by Mullin (2008). On the other hand, the majority of the theoretical investigations of pipe flow transition have been concerned with fully developed Hagen-Poiseuille flow, as discussed by Kerswell (2005) and Eckhardt et al. (2007). The central issue is that Hagen-Poiseuille flow is widely-accepted to be stable to infinitesimal perturbations (e.g. Meseguer \& Trefethen 2003) and yet, in practice, most pipe flows are turbulent. The process whereby turbulence arises is still not understood even in outline, and given its history and practical importance, this problem has become the outstanding challenge of hydrodynamic stability theory. The engineering implications of understanding transition in pipe flows are widespread, most notably in determining how large a pipe and how great a pressure gradient are needed to achieve a specified flow rate.

We will first review some widely-accepted facts about the stability of HagenPoiseuille flow and then discuss advances made this century on the topic. Hagen- 
Poiseuille flow takes $\approx R e / 30$ diameters (Fargie \& Martin 1971) to develop from a uniform inlet flow into a pipe, and the stability characteristics of this spatiallyevolving flow have attracted fewer investigations (as reviewed by Duck 2005) than the fully developed case. In carefully controlled experiments, developed HagenPoiseuille flow can be maintained up to $R e \sim 100,000$ (Pfenniger, 1961). Hence if it were possible to carry out a noise-free experiment in a perfectly circular, very long pipe, all evidence suggests that the flow would be laminar. A natural consequence of this is that, being dependent on both amplitude and form of the initial disturbance, there is no well-defined critical value of $R e$ for transition to turbulence. At low or 'transitional' $R e$ a more meaningful question is to ask whether a critical value $R e_{\text {low }}$ exists, below which turbulence cannot be maintained, i.e. indicating the transition from turbulence of a disturbance to the laminar state. The only theoretical result available is Joseph \& Carmi's (1969) energy stability result of 81.49 below which all disturbances are guaranteed to decay monotonically. This strict lower bound is, however, very conservative given experimental evidence places $R e_{\text {low }}=O(2000)$.

Transition from laminar flow is a result of finite amplitude disturbances either intentionally introduced or naturally present in the experiment and thus explains the wide range of values of $R e_{\text {low }}$ quoted in the literature (Mullin 2008). This sensitivity naturally poses a series of questions, such as which disturbance is the most dangerous? (i.e. triggers turbulence with the minimal energy or amplitude.) And how does this threshold amplitude or energy scale with increasing Re? The linear mechanism of transient growth is an important ingredient in the answers to these questions and was the focus of several studies at the end of the last century (Trefethen et al. 1993, Schmid \& Henningson 1994 and the review by Grossman 2000).

When transition occurs, it is generally abrupt and the character of the state achieved is $R e$-dependent. For $1760 \lesssim R e \lesssim 2,300$, localised 'puffs' appear as identified by Wygnanski\& Champagne (1973). A flow visualization image of a puff is shown in comparison with a numerical realization at $R e=1800$ (see figures 1 and 2). These are generally around $20 D$ long and have a weak front with a sharp trailing edge. They contain rich structure and maintain their form as they propagate along the pipe at approximately $0.9 U$. There is a lower bound in $R e$ for their existence where puffs can appear to suddenly decay without warning after travelling many hundreds of pipe diameters downstream. This meta-stability of puffs has undoubtedly contributed to the uncertainty surrounding the minimal $R e_{\text {low }}$ for sustained turbulence.

For $R e \gtrsim 2,700$, the disordered motion takes the form of 'slugs' Wygnanski et al. (1975). These are regions of fully turbulent flow which have sharp front and rear interfaces with the laminar flow field. The leading edge travels faster than the mean flow, the trailing edge slower, and so slugs expand as they propagate along the pipe. For $R e \gtrsim 10,000$ the leading and trailing edges travel at $\approx 1.5 U$ and $0.3 U$ respectively so that turbulence rapidly spreads along the pipe. The transition between puffs and slugs takes place in the $R e$ range $\approx 2300-2700$ and is complicated since it may involve puff splitting as discussed by Wygnanski et al. (1975). This general scenario was established by Wygnanski's group using entry flow disturbances in an air-flow pipe driven by a blower with a constriction which minimized pressure variations in the pipe when transition occurred. The finding have been confirmed 


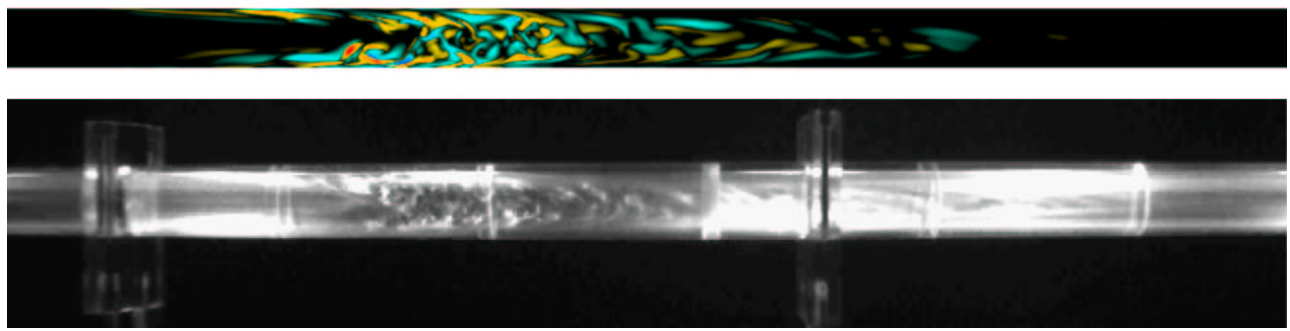

Figure 1. Experimental flow visualization (using Mearlmaid Pearlessence illuminated by a vertical sheet of light) compared with axial vorticity for a numerically calculated puff at the same $R e=1800$. The numerical resolution is 65 radial points, \pm 64 azimuthal Fourier modes and \pm 1024 axial Fourier modes, representing 34 million degrees of freedom.

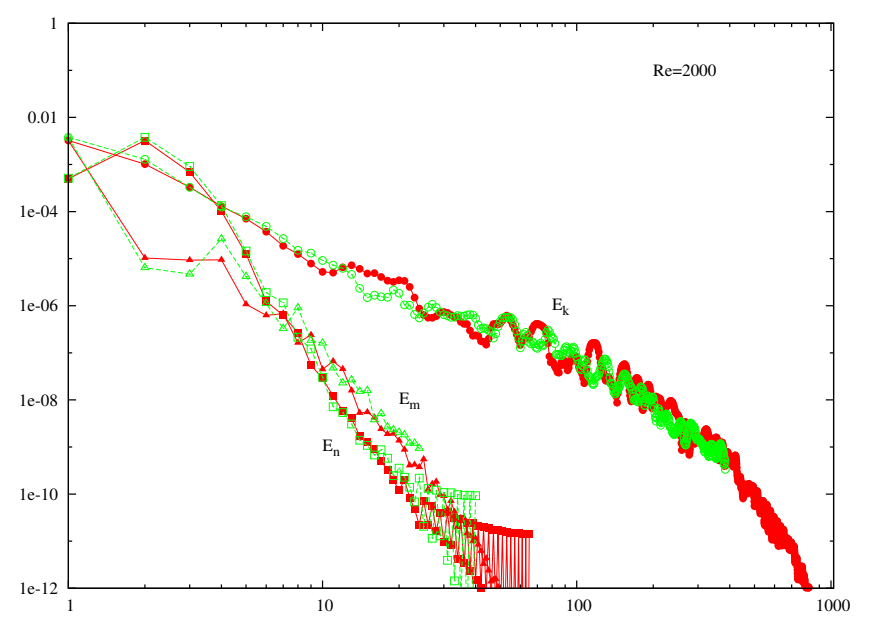

Figure 2. Numerical puff spectrum at $R e=2000, E_{n}=\max _{k m}\left[\max \left(u_{k m}^{2}, v_{k m}^{2}, w_{k m}^{2}\right)\right]$ where $\left(u_{k m}, v_{k m}, w_{k m}\right)$ are the radial, azimuthal and axial velocity spectral amplitudes with indices $n, m$ and $k$ referring to the (transformed) Chebyshev, Fourier and Fourier expansions in $(r, \theta, z)$, respectively (similarly for $E_{m}$ and $\left.E_{k}\right)$ : see Willis \& Kerswell (2007) for more detail. A snap shot from a run with $(n, k, m)$ up to $(40, \pm 24, \pm 384)$ (as in Willis $\&$ Kerswell 2007, 2008a) is compared with a snapshot taken from a much higher resolution of $(65, \pm 64, \pm 1024)$ run to show convergence.

for disturbances created in fully developed flow under constant mass flux conditions by Darbyshire \& Mullin (1995).

A significant recent theoretical development in pipe flow has been the discovery of the first alternative solutions to the unidirectional, steady Hagen-Poiseuille flow in the problem. These take the form of travelling waves (TWs) which appear through saddle node bifurcations for $R e \geq 773$ with upper (high wall shear stress) and lower branch (low wall shear stress) solutions and are immediately unstable, although intriguingly, only in a very small number of directions in state space. They were first found by searching within discrete rotational symmetry subspaces (Faisst \& Eckhardt 2003, Wedin \& Kerswell 2004) but later, TWs with no rotational symmetry were also isolated (Pringle \& Kerswell 2007). Each TW family is 


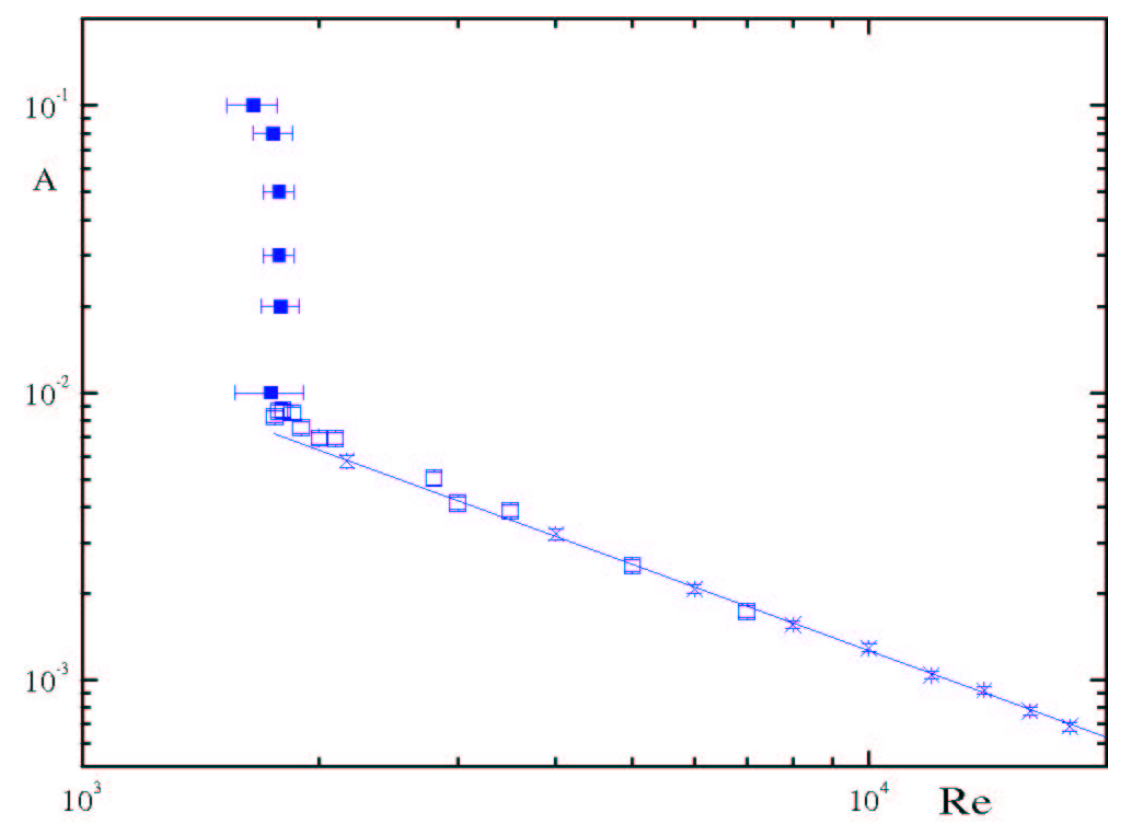

Figure 3. Plot of $A$ versus Re for six azimuthal jets, each with diameter $d=0.5 \mathrm{~mm}$. Disturbances with amplitudes above the threshold gave rise to puffs, slugs or turbulence downstream. Those below the line decayed within $100 \mathrm{D}$. Each point represents a minimum of 40 runs of the experiment. The filled symbols denote a lower bound of $R e$ for stable puffs. They were obtained by keeping $A$ constant and observing whether puffs relaminarised within the length of the pipe. The data points marked with a X are from Hof et al. (2003) and those with $\square, \boldsymbol{\square}$ are from Peixinho and Mullin (2007).

parameterized by its axial wavenumber which, at a given $R e$, has finite range (e.g. see figures $3 \& 4$ of Kerswell 2005). The significance of these solutions is that they present saddle points in phase space whose stable and unstable manifolds can tangle with each other to create a composite object (either a chaotic saddle or attractor) which is able to sustain orbits away from the laminar state for long times. At least initially, the hope was that the emergence of these solutions as $R e$ increases would provide a good estimator for transition. However rotationally-asymmetric TWs have now been found down at $R e=773$ (Pringle \& Kerswell 2007) which is under 50\% of $R e_{\text {low }}$. Exactly, why transition is delayed so long in $R e$ remains an intriguing issue.

The outline of this article is as follows. In section 2, we discuss results on threshold amplitudes of different disturbances needed to trigger transition. Experimental and numerical results on the relaminarisation problem are described in section 3 . Evidence collected thus far on the significance of TWs in transition is summarised in section 4 , and section 5 provides a brief perspective on what we consider to be some of the outstanding issues.

\section{Triggering Turbulence: Amplitude Thresholds}

As early as 1883, Reynolds realised that a finite amplitude disturbance is required to trigger transition and that the laminar flow becomes more and more sensitive to 
background disturbances as $R e$ increases. In order to establish whether the threshold for transition depends systematically with $R e$, control of the disturbance needs to be established. The exact positioning of the disturbance is also important as a distinction needs to be drawn between disturbances added to the inlet and those introduced into developed flow. Adding disturbances to developing flow is important for practical applications but making contact with theory is difficult since there is complex interaction between the developing base flow and any added disturbance. Binnie \& Fowler (1947) showed that these interactions could produce surprisingly long transient effects. Hence we focus here on the effects of adding finite amplitude perturbations to the well-defined case of fully-developed Hagen-Poiseuille flow.

Darbyshire \& Mullin (1995) established the possibility of systematic dependence of the threshold amplitude for disturbances added to fully developed flow. However, a scaling could not be extracted from their results and it was Hof et al. (2003) who showed that the threshold amplitude $A$ (defined by the mass flux) of a synchronised 6 -azimuthal jet disturbance has a scaling of $A \sim R e^{-1}$ over a significant range. These results are reproduced in figure 3 where additional data points have been added from recent work by Peixinho \& Mullin (2007) who also showed that a disturbance created by a single jet scales as $R e^{-1}$. Disturbance amplitudes which are below the threshold decay as they propagate downstream. The decay was normally complete within $100 \mathrm{D}$. For disturbance amplitudes above the threshold, transition took place within $100 \mathrm{D}$ and the state achieved was either a puff or slug depending on $R e$. Each data point was obtained by repeating the experiment around 40 times since the threshold is a statistical process as discussed by Darbyshire \& Mullin (1995). The left-hand set of points which are nearly vertical illustrate that there is an extreme dependence of the disturbance amplitude on $R e$, now appreciated to be related to the transition from turbulence. This is discussed in the following section, where an alternative strategy was required to explore this region systematically.

The key feature of the experiment used to obtain the results shown in figure 3 was to use a boxcar-shaped disturbance which allowed the amplitude and timescales of the applied disturbance to be separated. Recently, Peixinho \& Mullin (2007) have revisited this experiment using a 'push-pull' disturbance in the form of a jet and outlet pair which ensures that no net mass is added. Careful orientation of this jet and outlet with respect to the pipe axis was found to excite streaks and hairpin vortices. The threshold amplitude scalings were found to vary significantly from $A \sim R e^{-1}$ with scalings of $A \sim R e^{-1.3}$ when orientated in a spanwise or streamwise sense and $A \sim R e^{-1.5}$ for $45^{\circ}$ oblique orientation to the streamwise direction. Significantly, the amplitude thresholds for the push-pull disturbances were an order of magnitude less than those for jets implying that transition can be triggered much more easily by oblique vortices than jet disturbances. The exponents indicate that transient growth effects may be relevant and this is supported by observations of sequential transition sequence through streaks and hairpins. The creation of a hairpin vortex sequence has recently been confirmed numerically by Asen et al. (2008). In summary, crossing the divide between laminar and turbulent flows is generally a catastrophic process but careful design of the disturbance can reveal a more gradual transition where stronger contact with numerical simulations can be made.

Numerically, the challenge in making contact with experiments is replicating details of the spatially-localised nature of the initial disturbance applied in the lab- 


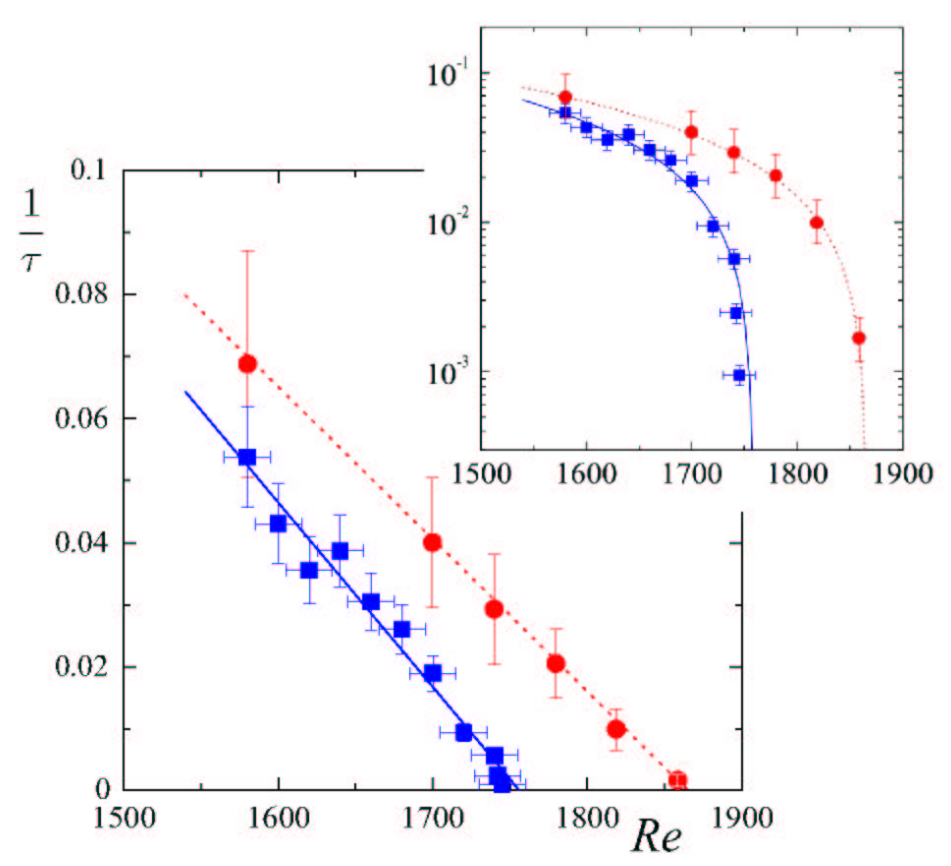

Figure 4. The mean half life $\tau$ plotted against $R e$ for experimental data (blue squares; from Peixinho \& Mullin 2006) and numerical simulations (red bullets; from Willis \& Kerswell 2007) for linear-linear and log-linear (inset) axes. The error bar for the numerical data point at $R e=1860$ on the main plot is smaller than the actual symbol.

oratory. Testing when turbulence is triggered by a global smooth disturbance is a more natural first step. Mellibovsky and Meseguer (2006) have examined the stability of pairs of axially-independent streamwise vortices to disturbances periodic along the axis. They obtained the scaling laws $A \sim R e^{-1}$ for 3 pairs, $A \sim R e^{-1.1}$ for 2 pairs and $A \sim R e^{\gamma}$ where $-1.5 \leq \gamma \leq-1.35$ for a lone pair of vortices. Subsequently, they have developed an ingenious localised body forcing to simulate a 6 -fold jet disturbance and find results which agree with Hof et al. (2003) for Re $>4000$ (Mellibovsky \& Meseguer 2007). This variability of scaling threshold with exact disturbance structure has been emphasized recently by other calculations exploring the flow dynamics artificially confined to the laminar-turbulent boundary. This boundary separates initial conditions which immediately laminarise from those which become turbulent. An initial condition placed precisely in this boundary will then by definition do neither, instead walking an interesting 'dynamical' tightrope between these two scenarios. The large-time behaviour of trajectories on this boundary is chaotic (Schneider et al. 2007), typical energies of which give another estimate of the threshold energy for transition (e.g. see figure 4 from Schneider et al. 2007 and figure $8 b$ of Willis \& Kerswell 2008b, which finds an $A \sim R e^{-1.5}$ scaling). Many travelling waves are found to be embedded in this boundary (Duguet et al. 2008). The direction from which this long-term behaviour within the boundary is approached, which may include an initial period of significant growth, explains the variation in scaling laws seen. The closest point of approach (in energy norm) of the laminar-turbulent boundary to the laminar state defines the most dangerous initial condition of all. 


\section{Relaminarisation: Threshold Re for Sustained Puffs}

Regardless of the exact structure of the disturbance, the amplitude thresholds show a divergence at $R e$ small enough (e.g. see figure 3), indicating that below a threshold $R e_{\text {low }}$ turbulence cannot be sustained. Identifying this threshold directly in experiments by adding very large disturbances is fraught with difficulty since significant distortion of the mean flow can result as discussed by Mullin \& Peixinho (2005,2006). Peixinho \& Mullin (2006) developed an alternative experimental protocol in which the relaminarisation of turbulence was considered for $R e<R e_{\text {low }}$. They created a puff of the type shown in figure 1 for $R e>R e_{\text {low }}$ in fully-developed flow using a well-defined disturbance. The puff was allowed to reach a developed 'equilibrium' state, before $R e$ was reduced in a controlled way to a selected value below $R e_{\text {low }}$. The statistics of how far the puff propagated before relaminarising was recorded over a large number of runs at the same Re. The probability of a puff lasting to a given distance was found to decay exponentially on average indicating a memoryless process (the probability of a puff relaminarising in a given distance or time interval depended only on the size of the interval). A well-defined half-life $\tau$ could be extracted for this process which satisfied the remarkably simple scaling relation $\tau \sim(1750-R e)^{-1}$, predicting that puff turbulence became sustained at $R e_{\text {low }}=1750$. This is in accord with estimates of $R e$ at which large amplitude perturbations do not give rise to transition downstream using a selection of different disturbances.

A notable advantage of this experimental protocol is that the procedure can be repeated using direct numerical simulations since the dependence on initial conditions has been effectively removed. Numerical investigations were carried out in a pipe $50 \mathrm{D}$ long across which periodic boundary conditions were applied, using over 3 million degrees of freedom and observing simulated puffs for times of up to $1000 \mathrm{D} / U$ (Willis \& Kerswell 2007). Random snap-shots from a long puff evolution generated at $R e=1900$ were used to initiate a series of puff runs at a given $R e<1900$. The same exponential probability distribution emerged as in experiments with the mean half life scaling like $\tau \sim(1870-R e)^{-1}$ indicating an estimate for $R e_{\text {low }}$ only $7 \%$ above the experimental value: see figure 4 . Given the sensitivity of the relaminarisation process and the different errors influencing the experiments and numerics, this correspondence, the first between theory and experiment in this problem, represents a significant success.

\section{Coherent Structures in Pipe Turbulence}

The initial discovery of TWs in pipe flow (Faisst \& Eckhardt 2003, Wedin \& Kerswell 2004) was closely followed by the first experimental sightings using PIV measurements in puffs and slugs (Hof et al. 2004). Subtracting the equivalent laminar state from the instantaneous flow field, clearly showed periodically-arranged fast streaks near the pipe wall and, at least for the puff cross-sections, interior slow streak structures which resembled those of the TWs (see figure 2 of Hof et al. 2004). The fact that these waves could appear, albeit transiently, as coherent structures in a turbulent flow was a landmark realisation in the shear flow community that unstable solutions could be dynamically relevant. From a dynamical systems standpoint, this was merely emphasizing that saddle points can be just as impor- 


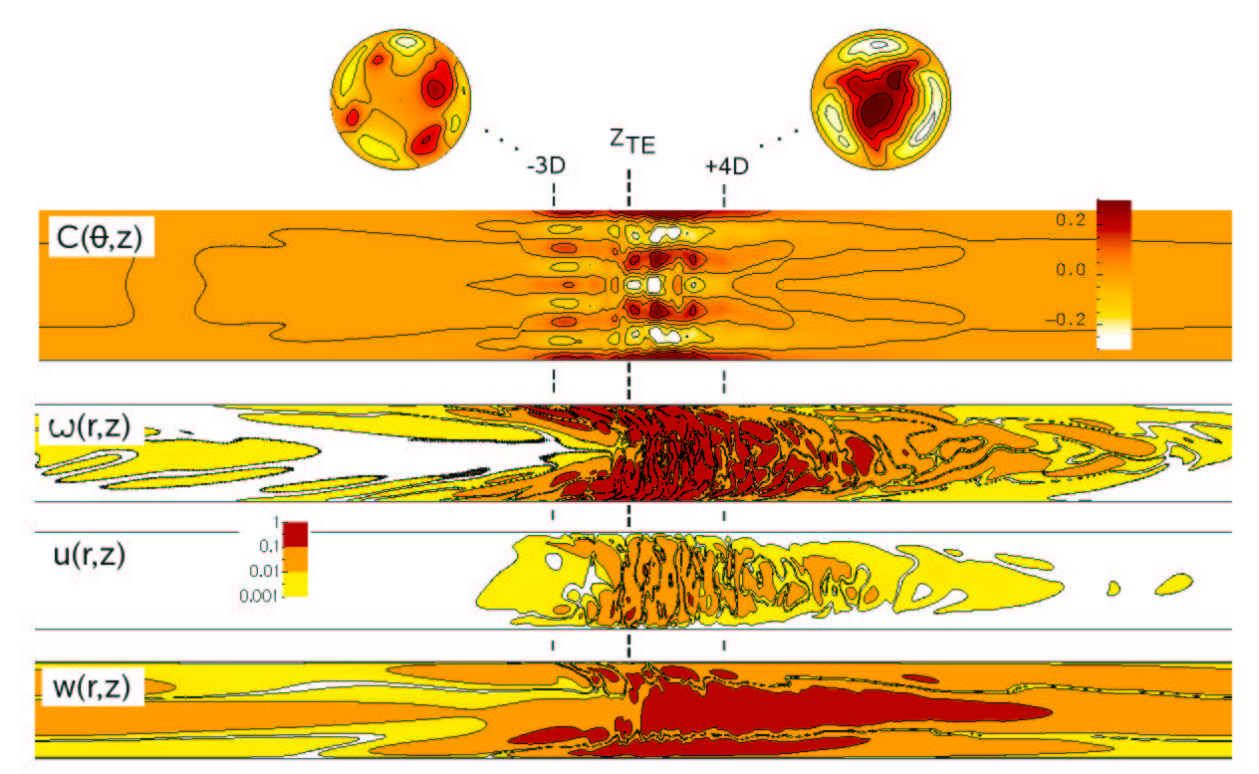

Figure 5. Appearance of TW-like structures upstream and downstream of the trailing edge $z_{\mathrm{TE}}$ in a snap-shot of a puff. In the cross-sections three- and four-fold rotationally symmetric flow fields are found at $z=z_{\mathrm{TE}}+4 D$ and $z=z_{\mathrm{TE}}-3 D$. Contours are of the streamwise velocity anomaly relative to the laminar flow: red(dark)/white indicating slower/faster moving fluid. Directly underneath is the contour plot of the correlation function $C(\theta, z):=2\left\langle u_{z}^{\prime}(\theta+\phi, z) u_{z}^{\prime}(\phi, z)\right\rangle_{\phi} /\left\langle\max _{\phi, z}\left(u_{z}^{\prime}\right)^{2}\right\rangle_{t}$ evaluated at $r=0.4 D$, where $u_{z}^{\prime}$ is the deviation from the time-averaged profile calculated at each $z-z_{\mathrm{TE}}$ station; shown over the full periodic $50 \mathrm{D}$ domain. $\langle\cdot\rangle_{s}$ indicates averaging over the subscripted variable. $C(\theta, z)$ is used to identify coherent fast streak structures. Below this are $(r, z)$-sections of the axial vorticity $\omega$, the radial velocity $u$ and the streamwise velocity difference $w$ from the laminar flow. Contours are at $0.001,0.01$ and 0.1 in units of $U / D(\omega)$ or $U(u, w)$ with the darkest colour indicating data $>0.1$ and white data $<0.001$. The plots show that $\omega$ and $u$ decay much more quickly than $w$. The decay of the azimuthal velocity component (not shown) is like that of $u$.

tant as attracting fixed points in organising phase space. Follow-up experimental work by Hof et al. (2005) in turbulent puffs concentrated upon finding evidence for the 'self-sustaining cycle' (Waleffe 1995,1997). This is the name given to the process whereby streamwise rolls, streaks and wavelike structures symbiotically coexist feeding energy to, and receiving energy from, each other and underpins the existence of the TWs (Wedin \& Kerswell 2004).

Attempts at quantifying how closely and how frequently TWs were approached by turbulent pipe flows came later utilising short $5 \mathrm{D}$ long pipe numerical simulations. Kerswell \& Tutty (2007) used a projection approach whereby the instantaneous flow field was projected in turn onto the velocity fields of all known TWs (prior to Pringle \& Kerswell 2007) in a pipe of that length. Good 'overlaps' indicating near-realisations of a TW were found to occur for $\approx 10 \%$ of the time. Schneider et al. (2007a) examined a streamwise velocity correlation function evaluated near the pipe wall to identify fast-streak coherent structures finding a higher incident rate consistent with a more inclusive criterion. While initiating their turbulent runs, 


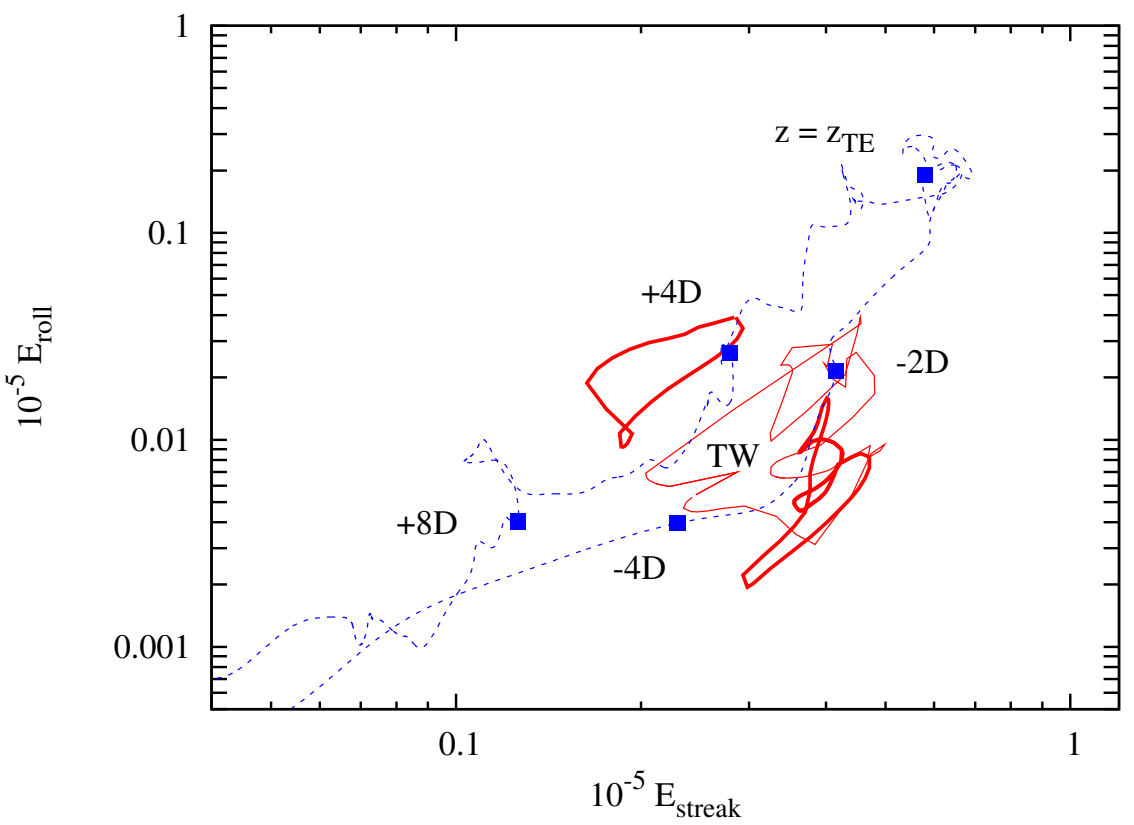

Figure 6. Roll and streak energies at different parts of a puff at $R e=2000$. For the velocity field expanded in Fourier modes $m$ in $\theta$, the $z$-dependent total streak and roll energies (blue dashed) are defined as $E_{\text {streak }}(z, t):=\pi R e^{2} \sum_{m \neq 0} \int\left|u_{m z}^{\prime}\right|^{2} r \mathrm{~d} r$ and $E_{\text {roll }}(z, t):=\pi \operatorname{Re}^{2} \sum_{m \neq 0} \int\left(\left|u_{m r}^{\prime}\right|^{2}+\left|u_{m \theta}^{\prime}\right|^{2}\right) r \mathrm{~d} r$, units $\rho \nu^{2}$. Here $\vec{u}^{\prime}=\left(u_{r}^{\prime}, u_{\theta}^{\prime}, u_{z}^{\prime}\right)$ is the deviation from the laminar profile, for easier comparison with the exact TW solutions (red solid lines). Energies for known travelling waves, marked 'TW', are parameterised by the continuum of axial wavenumbers permitted, are fairly low. The 3 'TW' lines correspond to waves with 2-fold, 3-fold and 4-fold discrete rotational symmetry about the axis (lower thick, thin middle and upper thick lines respectively).

Kerswell \& Tutty (2007) discovered that all the lower branch TWs they tried sat in the laminar-turbulent boundary: using the TW perturbed slightly along its most unstable direction led to a turbulent evolution whereas perturbing in the opposite sense led immediately to smooth relaminarisation. They also found that some of the upper branch TWs sat within the turbulent attractor at $R e=2400$, according to kinetic energy and wall shear-stress measures.

Further numerical work in $5 D$ pipes has recently focused on the laminar-turbulent boundary with Schneider et al. (2007) finding a chaotic attractor there in which the flow on average has a fascinatingly simple structure: two fast streaks sandwiching a slow streak near the pipe wall. This was later identified as an asymmetric TW (Pringle \& Kerswell 2007) which appears to represent the minimal coherent unit which can exist. This TW was found to bifurcate from a mirror-symmetric counterpart born at $R e=773$, considerably lower than the $R e=1251$ threshold found earlier for TWs with discrete rotational symmetry (Faisst \& Eckhardt 2003, Wedin \& Kerswell 2004). Work by Duguet at al. (2008) examining trajectories on the laminar-turbulent boundary has found evidence for repeated visits to other new TWs and heteroclinic connections between TWs likely to support complicated dynamics. 


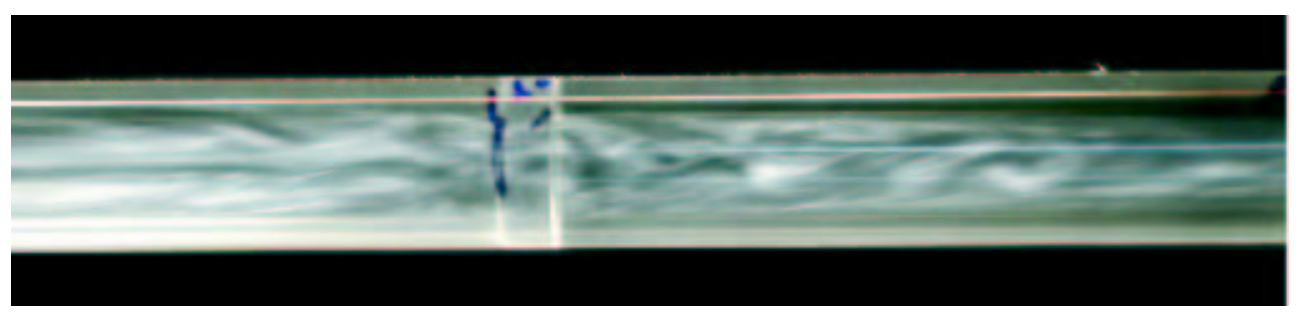

Figure 7. Flow visualization of wave at $R e=1740$ after reduction in $R e$ from 1900.

In longer pipes, Willis \& Kerswell (2008a) have used a 2-space 1-time streamwise correlation function to search for evidence of fast streak coherent structures in turbulent puffs. A significant subset of observed coherence bear a strong resemblance to travelling wave structures in regions upstream and downstream of the trailing edge (at $z_{T E}$ ), i.e. away from the most turbulently energetic area of the puff; an example from the calculation with 34 million degrees of freedom is shown in figure 5. Examining the partitioning of the energy into the roll and streak fields shows that there is near equipartitioning of energy between these two structures at the trailing edge whereas the defining characteristic of the TWs (both upper and lower branches) is the fact that the roll energy is typically at least an order of magnitude smaller than the streak energy; see figure 6 . The conclusion drawn from this is that at $R e=2000$ the TWs sit in an intermediate region of phase space removed from the turbulence, realised locally at $z_{T E}$. As a puff travels at only $\approx 0.9 U$, fluid, on average, passes through it. Far upstream $\left(z-z_{T E} \rightarrow-\infty\right)$, the fluid 'trajectory' starts at the origin (laminar state), passes through the TW-region of phase space as $z$ increases, to reach the fully turbulent region near the trailing edge. Downstream of the trailing edge, the trajectory passes back through the TW region to relaminarise as $z-z_{T E} \rightarrow+\infty$. Consequently, TWs are only visited just up- and down- stream of the trailing edge: see figure 6 for a 2-dimensional realisation of this. This picture of travelling waves being visited on relaminarisation resonates with some experimental observations reported by Peixinho \& Mullin (2006). During their relaminarisation experiments, they sometimes noticed transient wavelike structures downstream of the trailing edge. A flow visualization of a transient state at $R e=1740$ is shown in figure 7 . The evident wavelike structures were realized in $\approx 10 \%$ of the experimental runs and they have the characteristic form of a travelling wave with 2 -fold discrete rotational symmetry and a wavelength of $1.5 D$.

Most recent experimental evidence for the existence of travelling waves is provided by the investigations of Peixinho \& Mullin (2007). Using a push-pull disturbance aligned obliquely to the flow, they found a sequential onset process rather than the abrupt transition associated with the $R e^{-1}$ threshold found by Hof et al. (2003). Moreover, hairpin vortices were formed in the initial stages of transition for disturbance amplitudes close to threshold, and may be interpreted as TWs. An example of the creation of hairpin vortices during transition is given in the set of flow visualization images shown in figure 8 at $R e=2200$. The waves can clearly be seen in the top image which was taken 0.1 seconds after the push-pull disturbance was started. The propagating disturbance was tracked using a moving camera and the rapid breakdown to a puff can be seen. The trailing edge of the disturbance has only moved $\sim 4 D$ (0.6 secs) before significant disorder appears. 


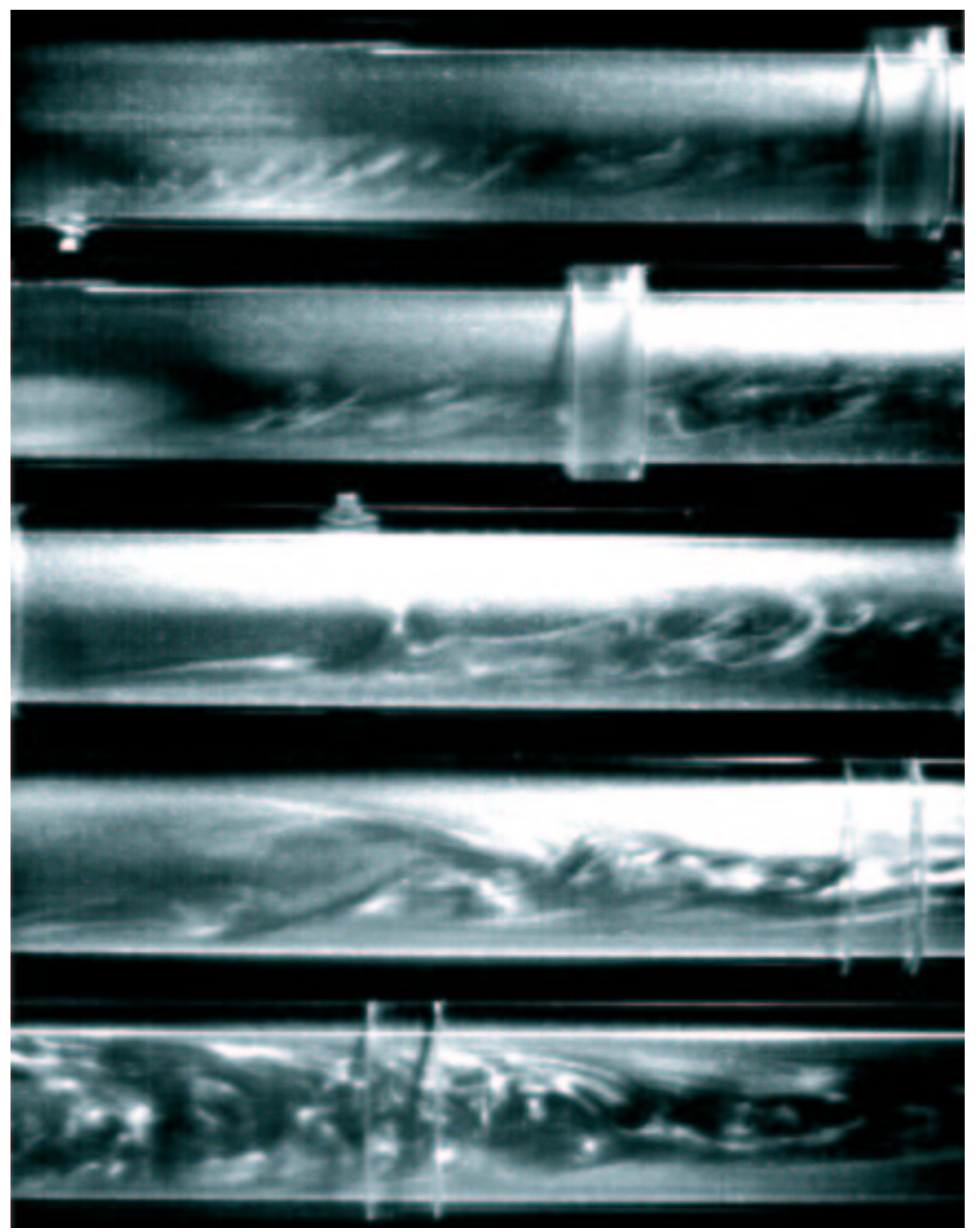

Figure 8. A sequence of flow visualization images showing the development of a disturbance from the oblique push-pull perturbation with $A=0.0023$ at $R e=2200$. The disturbance was injected in the plane of the photograph . Images were taken at $(a) 0.1,(b) 0.2,(c) 0.6$, $(d) 1$ and $(e) 1.5$ seconds after the beginning of the injection using a camera travelling at the same speed as the flow

\section{Outlook}

Despite the successes discussed above, many challenges still loom large. For example, why does a localised spatially-inhomogeneous turbulent structure which is the puff exist at all? How is the turbulence at the trailing edge able to remain localised? Is this region a valuable microcosm of global slug turbulence? And, of 
course, how can we characterise, predict and perhaps control the fully-developed turbulent state itself? The recent discovery of streaks and hairpin structures suggests that the strategy adopted by Fransson et al. (2006) for enhancing stability of laminar flow in boundary layers may be useful for pipe flows. The most dangerous part of the pipe for transition is the entrance region where disturbances can grow considerably before Hagen-Poiseuille flow is established. Controlling this using a grooved contraction to promote streamwise rolls and hence streaks may lead to control methods. As discussed above, Hagen-Poiseuille flow seems to be remarkably robust in practice and, hence, once it is established it is likely to be stable to disturbances of a predictable amplitude.

The phenomena described in this article all have plausible explanations using low-order dynamical systems theory. For example, the inferred global bifurcation of $\S 3$ at which transient puffs become sustained can be understood as a boundary crisis in reverse, that is, an attractor is born as $R e$ increases beyond the bifurcation point rather than being destroyed. Furthermore, the attractor itself and its leaky predecessor can be understood as the consequence of a tangle of homoclinic and heteroclinic connections between TW saddle points and other generic objects such as periodic orbits. At present, however, a step of faith has to be taken to make these connections. Pipe flow is, after all, the archetypal spatiotemporal system which specialises, at least at transitional $R e$, in selecting localised flows rather than global structures more immediately suited to a dynamical systems interpretation. Constructing the correct formalism to bridge this 'PDE to ODE' gap remains an outstanding challenge, not only in the pipe problem but in fluid mechanics as a whole.

Finally, it should hopefully be clear from this article that significant progress in the pipe flow problem has been recently achieved through the coordinated use of all three methodologies: experimentation, numerical simulation and theory. This balanced three-pronged attack will surely remain the ideal approach in exploring and understanding fluid mechanical phenomena in the 21st century.

The work was funded by EPSRC via grants GR/S76137/01 and GR/S76144/01 and the award of a Senior Research Fellowship to TM.

\section{References}

Asen, P.O., Kreiss, G. \& Rempfer, D. 2008 to appear in Theor. \& Comp. Fluid Dynamics. Binnie, A. M.\& J.S. Fowler 1947 A study by a double-refraction method of the development of turbulence in a long circular tube. Proc. Roy. Soc. A 192, 32-44.

Darbyshire, A.G. \& Mullin, T. 1995 Transition to turbulence in constant-mass-flux pipe flow. J. Fluid Mech. 289, 83-114.

Duck, P.W. 2005 Transient growth in developing plane and Hagen Poiseuille flow. Proc. Roy. Soc. A 461, 1311-33.

Duguet, Y., Willis, A.P. \& Kerswell, R.R. 2008 Transition in pipe flow: the saddle structure on the boundary of turbulence. J. Fluid Mech. submitted (http://arxiv.org/abs/0711.2175)

Eckhardt, B., Schneider, T. M., Hof, B., Westerweel, J. 2007 Turbulence transition in pipe flow. Ann. Rev. Fluid. Mech 39, 447-468.

Faisst, H. \& Eckhardt, B. 2003 Travelling waves in pipe flow. Phys. Rev. Lett. 91, 224502.

Fargie, D. \& Martin, B. W. 1971 Developing laminar flow in a pipe of circular cross-section. Proc. Roy. Soc. A 321, 461-476. 
Fransson, J.H.M., Talamelli, A., Brandt, L. \& Cossu, c. 2006 Delaying transition to turbulence by a passive mechanism Phys. Rev. Lett. 96, 064501.

Grossman, S. 2000 The onset of shear flow turbulence. Rev. Mod. Phys. 72, 603.

Hagen, G. H. L. 1839 Über die Bewegung des Wassers in engen cylindrischen Röhren. Poggendorfs Annalen der Physik und Chemie 16, 423

Hof, B., Juel, A. \& Mullin, T. 2003 Scaling of the turbulence transition threshold in a pipe. Phys. Rev. Lett. 91, 244502.

Hof, B., van Doorne, C.W.H., Westerweel, J., Nieuwstadt, F.T.M., Faisst, H., Eckhardt, B., Wedin, H., Kerswell, R.R. \& Waleffe, F. 2004 Experimental observation of nonlinear travelling waves in turbulent pipe flow. Science 305, 1594-1597

Hof, B., van Doorne, C.W.H., Westerweel, J. \& Nieuwstadt, F.T.M. 2005 Turbulence regeneration in pipe flow at moderate Reynolds numbers. Phys. Rev. Lett. 95, 214502.

Joseph, D.D. \& Carmi, S. 1969 Stability of Poiseuille flow in pipes, annuli, and channels. Quart. Appl. Math., 26, 575-599.

Kerswell, R.R. 2005 Recent progress in understanding the transition to turbulence in a pipe. Nonlinearity 18 R17-R44.

Kerswell, R.R. \& Tutty, O.R. 2007 Recurrence of travelling waves in transitional pipe flow. J. Fluid Mech. 584, 69-102.

Mellibovsky, F. \& Meseguer, A. 2006 The role of streamwise perturbations in pipe flow transition. Phys. Fluids 18074104.

Mellibovsky, F. \& Meseguer, A. 2007 Pipe flow transition threshold following localised impulsive perturbations. Phys. Fluids 19044102.

Meseguer, A. \& Trefethen, L. N. 2003 Linearized pipe flow to Reynolds number $10^{7}$. J. Comp. Phys. 186, 178-197.

Mullin, T. \& Peixinho, J. 2005 Recent observations on the transition to turbulence in a pipe. Proc. IUTAM conference on Transition to Turbulence, Bangalore.

Mullin, T. \& Peixinho, J. 2006 Transition to turbulence in pipe flow. J. Low Temp. Physics 145, $75-88$.

Mullin, T. 2008 Experimental investigations of transition to trubulence in a pipe. Appl. Mechs. Rev. (In preparation).

Peixinho, J. \& Mullin, T. 2007 Finite-amplitude thresholds for transition in pipe flow. J. Fluid Mech. 582, 169-178.

Peixinho, J. \& Mullin, T. 2006 Decay of turbulence in pipe flow. Phys. Rev. Lett 96, 094501.

Pfenniger W. 1961 Transition in the inlet length of tubes at high Reynolds numbers. In Boundary Layer and Flow Control (ed. G.V. Lachman), 970-980, Pergamon.

Poiseuille, J. L. M. 1840 Recherches expérimentelles sur les mouvement des liquides dans les tubes de très petits diamètres. C. R. Acad. Sci. 11, 961 \& 1041.

Pringle, C.C.T. \& Kerswell, R.R. 2007 Asymmetric, helical and mirror-symmetric travelling waves in pipe flow. 2007 Phys. Rev. Lett. 99, 074502.

Reynolds O. 1883 An experimental investigation of the circumstances which determine whether the motion of water shall be direct or sinuous and of the law of resistance in parallel channels. Proc. Roy. Soc. A 35, 84-99.

Schneider, T.M., Eckhardt, B. \& Yorke, J.A. 2007 Turbulence transition and the edge of chaos in pipe flow. Phys. Rev. Lett. 99034502.

Schmid, P.J. \& Henningson, D.S. 1994 Optimal energy density growth in Hagen-Poiseuille flow. J. Fluid Mech. 277197.

Schneider, T.M., Eckhardt, B. \& Vollmer, J. 2007a Statistical analysis of coherent structures in transitional pipe flow. Phys. Rev. E 75066313. 
Trefethen, L.N., Trefethen, A.E., Reddy, S.C. \& Driscoll, T.A. 1993 Hydrodynamic stability without eigenvalues. Science. 261, 578.

Waleffe, F. 1995 Hydrodynamic stability and turbulence: Beyond transients to a selfsustaining process. Stud. Appl. Maths. 95, 319-343.

Waleffe, F. 1997 On the self-sustaining process in shear flows. Phys. Fluids 9, 883-900.

Wedin, H. \& Kerswell, R.R. 2004 Exact coherent structures in pipe flow: travelling wave solutions. J. Fluid Mech. 508, 333-371.

Willis, A.P. \& Kerswell, R.R. 2007 Critical behaviour in the relaminarisation of localised turbulence in pipe flow. Phys. Rev. Lett. 98, 014501.

Willis, A.P. \& Kerswell, R.R. 2008a Coherent structures in localised and global pipe turbulence. Phys. Rev. Lett. 100, 124501.

Willis, A.P. \& Kerswell, R.R. 2008b Turbulent dynamics of pipe flow captured in a 2.5D model: puff relaminarisation and localised 'edge' states. (submitted, arXiv:0712.2739)

Wygnanski, I. J. \& Champagne, F. H. 1973 On transition in a pipe. Part 1. The origin of puffs and slugs and the flow in a turbulent slug. J. Fluid Mech. 59, 281-351.

Wygnanski, I.J., Sokolov, M. \& Friedman, D. 1975 On transition in a pipe. Part 2. The equilibrium puff. J. Fluid Mech. 69, 283-304. 